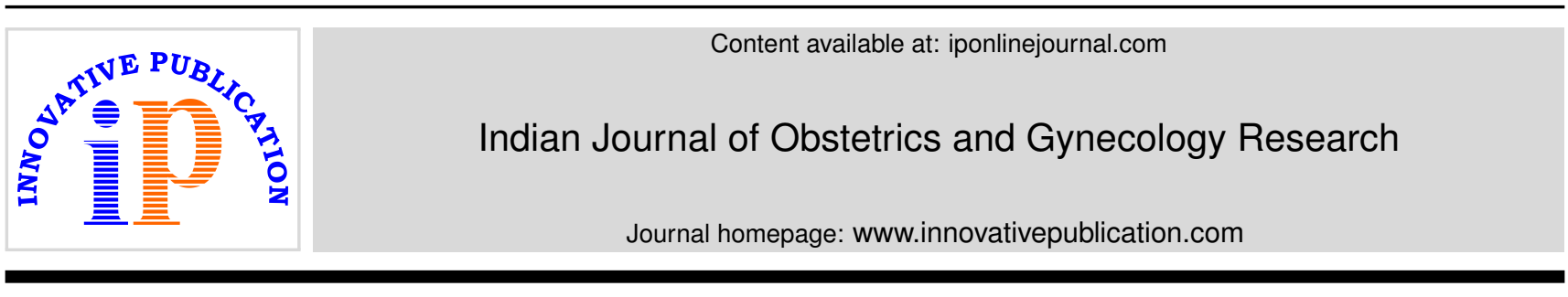

Original Research Article

\title{
Clinicopathological vaginal discharge among pregnant women: Pattern of occurrence and associated complications
}

\author{
Pooja Bhatt ${ }^{1}$, Vidyashree $\mathbf{R}^{1, *}$ \\ ${ }^{1}$ Dept. of Obstetrics and Gynaecology, Adichunchanagiri Institute of Medical Sciences, B.G Nagara, Karnataka, India
}

\section{A R T I C L E I N F O}

\section{Article history:}

Received 10-01-2020

Accepted 15-0-2020

Available online 15-06-2020

Keywords:

Candidiasis

Bacterial Vaginosis

Trichomoniasis

\begin{abstract}
A B S T R A C T
Introduction: Vaginal discharge is a common gynaecological condition among women of childbearing age that requires care. Pathological vaginal discharge can cause serious harm to pregnant women and the newborn.

Aims and Objectives: To determine the prevalence, pattern and risk factors associated with clinicopathological discharge and its complications.

Materials and Methods: A prospective study was done from January 2019 to August 2019 in Department of OBG at Adichunchanagiri Institute of Medical sciences, Bellur, Karnataka. The study included all cases of pregnant women with vaginal discharge.

Result: A total of 100 women attending antenatal clinic with white discharge were studied. Out of which $30(30 \%)$ women were symptomatic and $70(70 \%)$ asymptomatic. Out of $30(30 \%)$ symptomatic patients there were $23(76.6 \%)$ Vaginal Candidiasis, $6(20 \%)$ Bacterial Vaginosis and 1 (3.3\%) Trichomoniasis. Out of $30(30 \%)$ symptomatic patients there were $8(26.6 \%)$ developed threatened preterm, $6(20 \%)$ PROM, 2 $(6.6 \%)$ Preterm delivery. Out of 100 vaginal discharge patients there were $58(58 \%)$ primigravida and 42 (42\%) multigravida.

Conclusion: Pathological vaginal discharge in pregnant women is found to be most common in younger age group and in primigravida. Strong association has been found between pathological vaginal discharge and threatened preterm, PROM and prematurity. Our study concludes the need for timely detection and advise on good hygiene habits and intervention in the form of medical management and prevention of complications.
\end{abstract}

(C) 2020 Published by Innovative Publication. This is an open access article under the CC BY-NC license (https://creativecommons.org/licenses/by-nc/4.0/)

\section{Introduction}

Vaginal discharge is a common gynaecological condition among women of childbearing age that requires care. Pathological vaginal discharge can cause complications in pregnant women and the new born. Complications in the pregnant women such as Threatened preterm, premature rupture of membranes (PROM), Preterm labour, chorioamnionitis and post-partum endometritis.

Complications in new born are prematurity, low birth weight, respiratory distress. normally there is increased cervical secretions and vaginal transudate during pregnancy

\footnotetext{
* Corresponding author.

E-mail address: raysterganesh@gmail.com (Vidyashree R).
}

due to increased vascularity and hyper estrogenic state. Vaginitis due to candida albicans is relatively more common than other organisms most commonly patient presents with vaginal discharge, foul smell, itching, lower pain abdomen and rarely fever. Asymptomatic colonization of organisms requires no treatment. Hence screening is not recommended routinely. However symptomatic patients require prompt treatment.

\section{Aim and Objective}

To determine the prevalence, pattern and risk factors associated with clinico-pathological discharge and its complications. 


\section{Materials and Methods}

A prospective study was done from January 2019 to August 2019 in department of OBG at Adichunchanagiri Institute of Medical Sciences, B G Nagara, Karnataka. The study included all pregnant women with vaginal discharge.

Patients with vaginal discharge evaluated with the following

1. Clinical history

2. Blood investigatins such as $\mathrm{CBC}$, urine routine, RBS

3. High vaginal swab for culture and sensitvity

\section{Result}

Total of 100 women attending antenatal clinic with white discharge were studied.

Total no of cases-100. Total no of asymptomatic patients - 70 and symptomaticpatients- 30 .

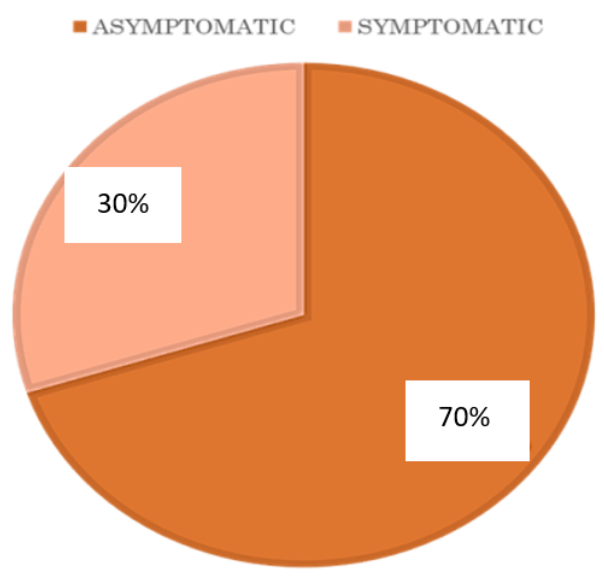

Fig. 1:

\subsection{Age distribution}

Out of 100 pregnant women 22 were $</=20$ years of age, 40 were between 21 to 25 years of age, 26 were between 26 to 30 years of age and 12 were between 31 to 35 years of age.

Out of 100 pregnant women who developed Vaginal discharge , $58(58 \%)$ were primigravida and $42(42 \%)$ were multigravida.

\subsection{Risk factors}

Out of 100 patients there were 76 from low socioeconomic status risk group, 14 were from urinary tract infection risk group, 47 were from anaemia risk group, 9 were from diabetes risk group and 10 were from vaginal discharge in previous pregnancy.

Incidence of vaginitis in symptomatic patients: Out of 30 (30\%) symptomatic patients there were 23 (76.6\%) Vaginal

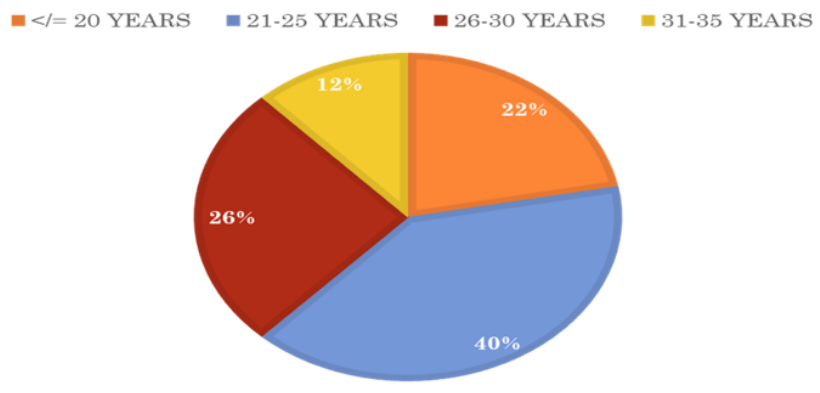

Fig. 2: Age distribution

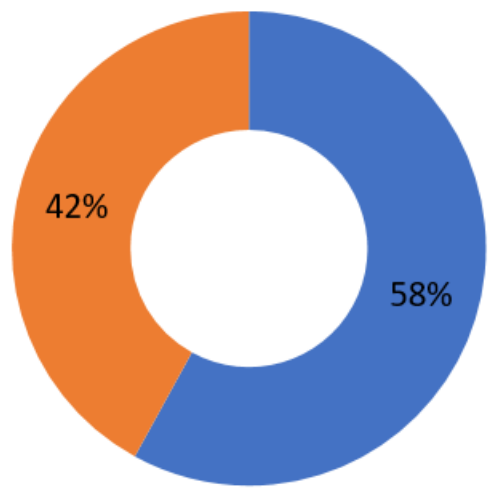

PRIMIGRAVIDA MULTIGRAVIDA

Fig. 3:

Table 1:

\section{Risk Factors}

Low socioeconomic

Urinary tract infection

Anemia

Diabetes

Vaginal discharge in previous

pregnancy

Candidiasis, 6 (20\%) Bacterial Vaginosis and 1 (3.3\%) Trichomoniasis.

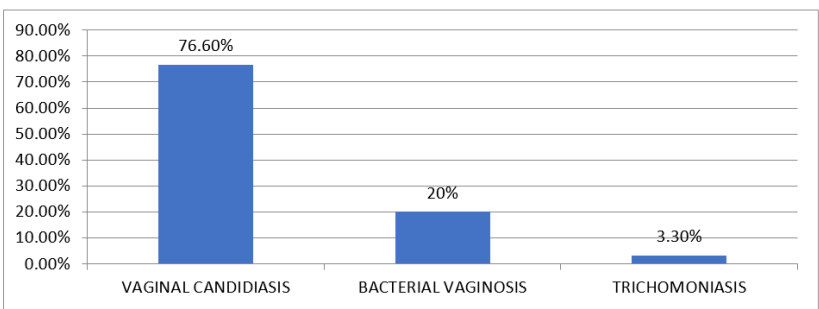

Fig. 4: 
Out of $30(30 \%)$ symptomatic patients there were 8 (26.6\%) developed threatened Preterm, 6 (20\%) PROM, 2 $(6.6 \%)$ Preterm delivery.

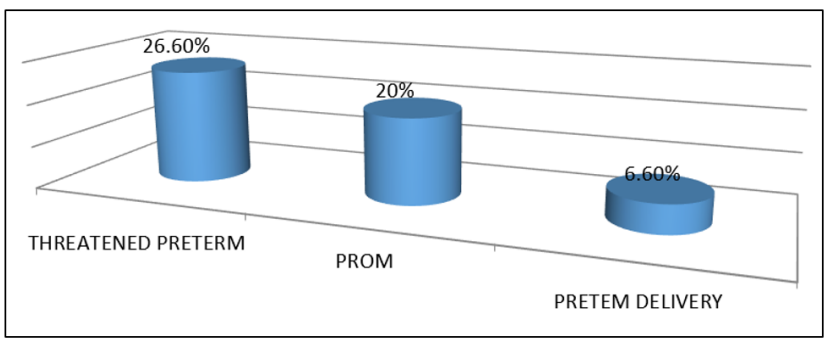

Fig. 5:

\section{Discussion}

In our study Vaginal discharge affected $30 \%$ of the women. A higher prevalence of vaginal discharge during pregnancy was seen in women younger than 24 .

Risk factors associated were young age, low socioeconomic condition, vaginal discharge in a previous pregnancy, anemia and urinary infection.

Hence timely detection and prompt treatment helps to avoid maternal and perinatal morbidity and improves the pregnancy outcome.

\subsection{In other study}

In a cross sectional study conducted by Tania Maria and et $\mathrm{al}^{1}$ in the city of Rio Grande, 2,395 pregnant women were included in the study.

The prevalence of vaginal discharge during pregnancy was $43 \%$ among all women studied. About $20 \%$ were adolescents (younger than 20), 44\% were primiparous and $11 \%$ reported pathological vaginal discharge in a previous pregnancy.

Pathological vaginal discharge during pregnancy was significantly associated with the following variables: maternal age; vaginal discharge in a previous pregnancy $(84 \%)$; diabetes $(45.3 \%)$ and urinary infection (54.2\%).

In a study conducted by Sanusi Mohammed Ibrahim and et $\mathrm{al}^{2}$ in the University of Maiduguri Teaching Hospital, Nigeria. 800 pregnant women were studied.

Vaginal discharge in pregnancy is common, but distinguishing abnormal vaginal discharge from normal leucorrhoea of pregnancy is challenging. Since findings have showed that the trio of vaginal candidiasis, trichomoniasis and bacterial vaginosis are common causes of abnormal vaginal discharge in pregnancy; efforts must be made to exclude these conditions in pregnant patients presenting with vaginal discharge so that appropriate treatment can be instituted timely.

Of the 400 patients with abnormal vaginal discharge, the commonest microorganism found was C. albicans,
$160(40 \%)$, whereas N. gonorrhoea infection was the least, $1(0.2 \%)$. E. coli was isolated in $20(5 \%)$, $\mathrm{T}$. vaginalis in $7(1.8 \%)$, Staphylococcus aureus in $36(9 \%)$, Klebsiella species in $8(2 \%)$, G. vaginalis in $68(17 \%)$ and Streptococcus species in $8(2 \%)$ of the pregnant women. Samples from $92(23 \%)$ patients had negative culture.

The high prevalence observed in this study of infections such as candidiasis, trichomoniasis, and bacterial vaginosis in pregnant women emphasizes the need to adopt effective strategies for their early detection associated with a reinforcement in the measures for prevention and treatment of both women and their partners.

In this study only the discharge among pregnant women and the organisms responsible for vaginal discharge has been studied.

\section{Conclusion}

Pathological vaginal discharge in pregnant women is found to be most common in younger age group and in primigravida.

Strong association has been found between pathological vaginal discharge and threatened preterm, PROM and prematurity.

Our study concludes the need for timely detection and advise on good hygiene habits and intervention in the form of medical management and prevention of complications.

\section{Abbreviations}

Candidiasis, Bacterial Vaginosis, Trichomoniasis.

\section{Source of Funding}

None.

\section{Conflict of Interest}

None.

\section{References}

1. da Fonseca TMMV, Cesar JA, Mendoza-Sassi RA, Schmidt EB. Pathological Vaginal Discharge among Pregnant Women: Pattern of Occurrence and Association in a Population-Based Survey. Obstet Gynecol Int. 2013;2013. त01:0. $155 / 2013 / 590416$

2. Ibrahim SM, Bukar M, Audu BM. Management of abnormal vaginal discharge in pregnancy; 2016. 101:10.5/72/62509.

\section{Author biography}

Pooja Bhatt Assistant Professor

Vidyashree R Postgraduate

Cite this article: Bhatt $P$, Vidyashree $R$. Clinicopathological vaginal discharge among pregnant women: Pattern of occurrence and associated complications. Indian J Obstet Gynecol Res 2020;7(2):193-195. 